\title{
The mortality patterns of lung cancer between 1990 and 2013 in Xuanwei, China
}

\author{
Gongbo Chen ${ }^{\mathrm{a}, \mathrm{b}}$, Xin Sun ${ }^{\mathrm{c}}$, Hongyan Ren ${ }^{\mathrm{d}}$, Xia Wan ${ }^{\mathrm{a}}$, Hecang Huang ${ }^{\mathrm{e}}$, Xiangyun Ma ${ }^{\mathrm{e}}$, \\ Bofu Ning ${ }^{\mathrm{e}}$, Xiaonong Zou ${ }^{\mathrm{b}}$, Weijiang $\mathrm{Hu}^{\mathrm{c}}$, Gonghuan Yang ${ }^{\mathrm{a}, *}$ \\ a Institute of Basic Medical Sciences, Chinese Academy of Medical Sciences E School of Basic Medicine, Peking Union Medical College, Beijing 100005, China \\ ${ }^{\mathrm{b}}$ Cancer Hospital/Institute, Chinese Academy of Medical Sciences \& Peking Union Medical College, Beijing 100021, China \\ c National Institute of Occupational Health and Poison Control, Chinese Center for Disease Control and Prevention, Beijing 100050, China \\ ${ }^{\mathrm{d}}$ Institute of Geographic Sciences and Nature Resources Research, Chinese Academy of Sciences, Beijing 100101, China \\ e Xuanwei Center for Disease Control and Prevention, Xuanwei, Qujing, Yunnan 655400, China
}

\section{A R T I C L E I N F O}

\section{Article history:}

Received 25 December 2014

Received in revised form 1 August 2015

Accepted 13 August 2015

\section{Keywords:}

Mortality patterns

Causes of death

Lung cancer

Xuanwei

\begin{abstract}
A B S T R A C T
Objectives: To explore the variations in the mortality trends, especially death due to lung cancer, from 1990 to 2013 in Xuanwei City.

Materials and methods: Mortality data were collected in Xuanwei during the 2nd and 3rd National Retrospective Sampling Survey on Mortality and Routine Death Registration System (DRS) during 2011-2013. According to the result of the survey on under-reported deaths, mortality data from DRS during 2011-2013 were adjusted. Disease specific mortality rate, age-standardized mortality rate (ASMR) and $45 \mathrm{Q} 15$ were calculated in Xuanwei and compared with those in rural areas of China.

Results: During three periods, 1990-1992, 2004-2005 and 2011-2013, lung cancer contributed to 56.86\%, $58.45 \%$ and $63.03 \%$ of deaths from all cancers respectively with a much higher proportion than rural areas nationally. The ASMR of lung cancer for males surged from $41.43 / 10^{5}$ to $88.17 / 10^{5}$ during $1990-2005$ and it surged from $37.70 / 10^{5}$ to $74.45 / 10^{5}$ for females. Although they declined slightly during $2011-2013$ $\left(82.53 / 10^{5}\right.$ and $62.62 / 10^{5}$ for males and females respectively), the ASMR of lung cancer among males in Xuanwei was three times of that in rural areas in China, and it was six times higher among females. The 45Q15 of lung cancer for males in Xuanwei was 3-5 times of that in rural areas of China and for females it was 7-9 times. The high-mortality areas of lung cancer were still located in Laibin, Longchang, Wanshui and Shuanglong Communities. High-mortality areas of lung cancer expanded to their surrounding areas and those in southeast.

Conclusions: Although indoor air pollution caused by smoky coal has been fairly well controlled, patterns of death due to lung cancer have not obviously changed. The mortality rate of lung cancer among females was similar to that among males. Therefore, further studies should be conducted to comprehensively explore the risk factors of lung cancer in Xuanwei.
\end{abstract}

(c) 2015 Published by Elsevier Ireland Ltd.

\section{Introduction}

According to a national retrospective survey on cancer mortality during 1973-1975, Xuanwei City in Yunnan Province was an area with almost the highest mortality rate of lung cancer in China, which was 5.3 times of that in rural areas nationally [1]. Agestandardized mortality rates (ASMR) were $23.14 / 10^{5}$ and $4.34 / 10^{5}$ for Xuanwei and rural areas in China, respectively. ASMRs for males and females in Xuanwei during 1973-1979 were 27.7/10

\footnotetext{
* Corresponding author.

E-mail address: yangghuan@vip.sina.com (G. Yang).
}

and $25.3 / 10^{5}$ respectively (age adjusted to 1964 China population), whereas they were $6.8 / 10^{5}$ and $3.2 / 10^{5}$ in rural areas in China [2]. Particularly in high-mortality communities (Chengguan, Laibin and Rongcheng), mortality rate of lung cancer among females was higher than that among males (mortality rate for males was $118 / 10^{5}$ and $125.6 / 10^{5}$ for females). Furthermore, regional differences were remarkable [2]. Previous studies have illustrated that the main cause of high mortality of lung cancer is indoor air pollution caused by the use of smoky coal and unimproved stoves [2-5]. Carcinogenic substances produced in the process of coal combustion are PAHs, including Benzopyrene [6], particulate matter and quartz [7]. Animal experiments have also demonstrated that combustion emissions are carcinogenic to mice [8]. In 1970s, the 
government encouraged and supported local residents to improve their stoves, and this stove improvement project was completed in the late 1980s. According to previous studies, indoor exposure to Benzopyrene and particulate matter, as well as the risk of lung cancer decreased after stove improvement $[9,10]$. However, the mortality rate due to lung cancer did not decline as expected $[11,12]$. Only using national surveys conducted during $10-20$ year intervals causes difficulties in comprehensively observing patterns of lung cancer. In 2006, the routine death registration system (DRS) was established in Xuanwei which provides feasibility to observe patterns of lung cancer continuously. In this paper, with mortality data extracted from the National Retrospective Sampling Surveys on Mortality and DRS, we explored variations in the total death patterns, especially mortality of lung cancer and compared them with rural areas of China.

\section{Materials and methods}

\subsection{Data sources}

(1) Mortality data during 1990-1992 and 2004-2005 were obtained from the 2nd and 3rd National Retrospective Sampling Survey on Mortality. The methods of survey and data collection in Xuanwei were the same as those used nationally [11,13].

(2) Mortality data during 2011-2013 were extracted from direct online reports of DRS in Xuanwei CDC. In regard to deaths that occurred at home or in hospitals, a death certificate or medical certificate of death were written by medical workers in communities or villages and doctors in hospitals, respectively. Public health physicians coded underlying causes of death using ICD-10 and report them to China CDC.

(3) Population data were obtained from three censuses and annual statistics of the population in Xuanwei City [14-16]. Population data for the whole city and every community in other years except for census were from the Department of Statistics and were dependent on the statistics of resident registration data [17].

\subsection{Quality of data}

Completeness of data was evaluated by report rate and reliability of diagnoses was evaluated by institutions making diagnoses, types of diagnoses and the proportion of deaths with unknown causes.

\subsubsection{Completeness of data}

The completeness of mortality data during 1990-1992 and 2004-2005 were evaluated and accepted in 2nd and 3rd National Retrospective Sampling Survey on Mortality [11,13]. Regarding mortality data from local DRS during 2011-2013, under-reported deaths exist since DRS was established in Xuanwei in 2006. To evaluate completeness of data from DRS, a survey on under-reported deaths was conducted in Xuanwei in August, 2014. Details of this survey were reported elsewhere [18]. In brief, the survey was conducted in 30 villages of 21 communities selected with a multi-stage random sampling strategy. A total of 13,960 families were interviewed covering $3.3 \%$ of the entire population. Capture-recapture method was employed to calculate under-reported rate of death [19]. Total under-reported rate of death was $31.88 \%$. In highmortality areas (96 out of 354 villages), the under-reported rate of death was $17.48 \%$, and in medium-mortality areas (206 villages) and low-mortality (52 villages) areas they were $38.01 \%$ and $36.22 \%$, respectively. Mortality data from DRS during 2011-2013 adjusted according to regional under-reported rates were included in our final analysis.

\subsubsection{Accuracy of diagnosis}

During 1990-1992, $41.09 \%$ of deaths were diagnosed at medical institutions at the level of county or above (district or province level). During 2004-2005 and 2011-2013, it was 54.50\% and $58.13 \%$, respectively (Table A.1 in appendices). Particularly, all records for lung cancer cases occurred in 2013 were further reviewed and checked by local medical workers. In 2013, $12.6 \%$ of lung cancer cases had surgery before their deaths, and $21.3 \%$ of them had sputum cytology or pathology examination including needle biopsy and bronchofiberoscopy. The remaining cases had chest CT scan or X-ray. Besides, almost $99 \%$ of lung cancer cases were diagnosed by city level or above hospitals in 2013 which is consistent with the proportion reported in our database (around 95\% during three periods), including $35.22 \%, 22.4 \%$ and $41.2 \%$ in province, district and city level hospitals respectively. Part of hospital records of lung cancer cases in two city level hospitals were reviewed by a clinical oncologist from the Cancer Hospital of Chinese Academy of Medical Sciences and a pathologist from Henan Cancer Hospital. According to their evaluation, diagnosis of cancers in hospitals in Xuanwei City was reliable, with coincidence rate between $86.7 \%$ and $90.9 \%$.

\subsection{Statistical analysis}

Mortality data were adjusted by garbage code redistribution and under-reported rates of death, and mapped from ICD9 to ICD10 owing to death cases during 1990-1992 coded with ICD-9 [20] and those after 2004 using ICD-10 [21]. We linked ICD-9 and ICD-10, which are classified as the same category and form unified death codes including 120 causes of death (Table A.2 in appendices). Deaths caused by senility, anemia, heart failure, pulmonary heart disease, et al., are identified as garbage codes in mortality data as they are not underlying causes. Considering the small proportion of garbage code $(3.96 \%, 1.40 \%$ and $4.53 \%$ during three periods respectively), we do not redistribute them into other categories except for deaths caused by pulmonary heart disease (ICD-9: 416, ICD10I27.9) which are classified as underlying cause of COPD, as most cases of pulmonary heart disease were developed from COPD in China [22]. The mortality rates were further adjusted by envelope multiplying crude death proportion by causes.

Age-specific populations in each village and community were estimated by multiplying the total population with the proportion of population in each age group. The mortality patterns of causes of death in Xuanwei City were compared with those in rural areas in China during the three time periods [11,23]. Because national data of DSP in 2013 have not been published, data for causes of death in Xuanwei during 2011-2013 were compared with data of DSP in China in 2012 [24].

Statistical indicators are calculated including number of deaths, crude mortality rate, life expectancy, constituent ratio, causespecific mortality rate, age standardized mortality rate (ASMR), 45Q15 (probability of death between age 15 and 60) [25] and confidence intervals for ASMR [26].

\section{Results}

\subsection{Age structure, total mortality rate and causes of death}

In Xuanwei, aged population accounted for an increasing proportion similarly to that nationally. The proportion of population aged 60 or above increased from 6.6\% to $11.1 \%$ during $1990-2010$. The total mortality rate among population in each age group for each gender is higher than that in rural areas nationally. During 2011-2013, life expectancy of Xuanwei population was 71. 63 years old which was 3.2 years lower than that in China. Similar to 


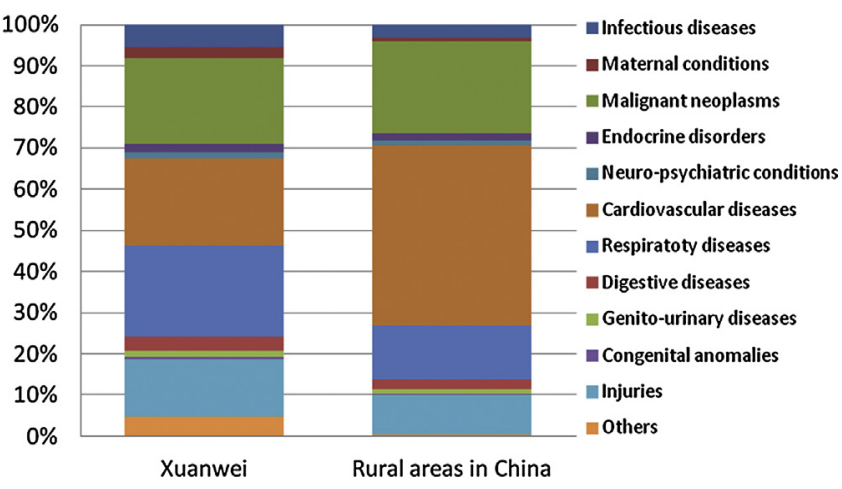

Fig. 1. Causes of deaths in Xuanwei and rural areas of China during 2011-2013.

rural areas of China, the proportions of deaths from communicable, maternal, perinatal and nutritional conditions continued to decline, while that of death from non-communicable diseases kept increasing. In terms of causes of death in each period in Xuanwei, the most noticeable characteristic was that respiratory disease accounted for a high proportion of death and the proportion of deaths due to malignant neoplasms was slightly higher than that in rural areas nationally (Details are shown in table A.3 in appendix). However, cardiovascular disease accounted for a lower proportion compared with that in rural areas nationally (Fig. 1). During 1990-2013, the proportion of deaths from respiratory disease declined, and that of deaths from malignant neoplasms and cardiovascular disease increased. However, the differences between constituent of death causes in Xuanwei and that in rural areas nationally remained unchanged.

\subsection{Variations in mortality due to lung cancer}

As a high-mortality area of lung cancer since 1970s, lung cancer remained the top cause of death from all cancers. During 1990-1992, 2004-2005 and 2011-2013, lung cancer accounted for $56.86 \%, 58.45 \%$ and $63.03 \%$ of all deaths from cancers, respectively, and the proportions were $57.73 \%, 60.66 \%$ and $66.70 \%$, respectively, among females, which were higher than those among males. However, lung cancer accounted for $25.40 \%, 20.94 \%$ and $25.85 \%$ of deaths from all cancers, respectively, in rural areas of China during those three periods. The constituent ratio of deaths from lung cancer among all cancers was more than two times of that in rural areas in China although it increased to $25.85 \%$ nationally during 2011-2013 (Fig. 2).

During 1990-1992, crude mortality rates of lung cancer in Xuanwei for males and females were $36.79 / 10^{5}$ and $31.00 / 10^{5}$, respectively. During 2004-2005, they increased to $96.33 / 10^{5}$ and $81.56 / 10^{5}$, respectively, and they reached $116.35 / 10^{5}$ and $92.00 / 10^{5}$, respectively, during $2011-2013$. During the past two

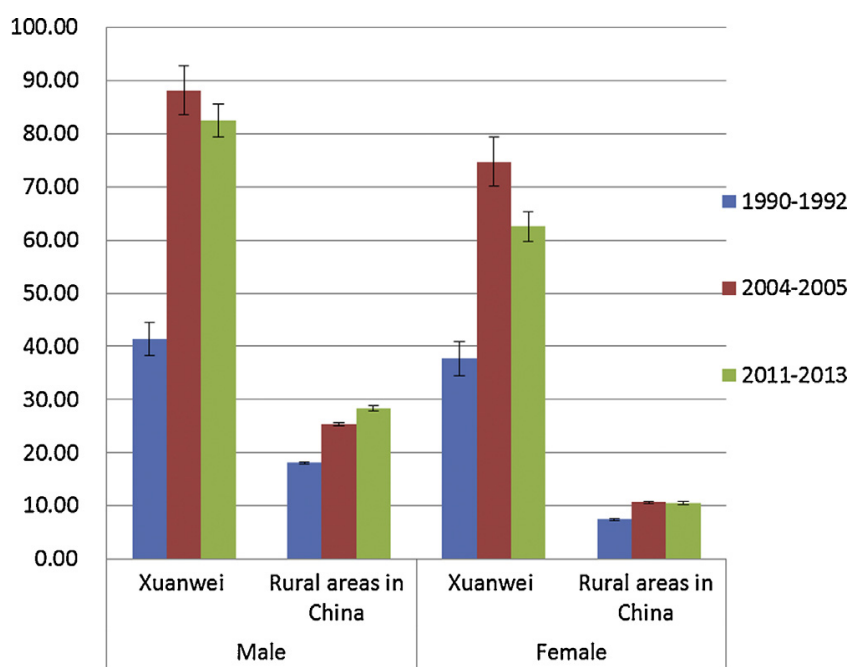

Fig. 3. ASMR of lung cancer in Xuanwei and rural areas of China during 1990-2013 $\left(1 / 10^{5}\right)$.

decades, the ratio of lung cancer mortality rates among males to that among females increased from 1.1 to 1.3. However, they were significantly different from that in rural areas of China, which were nearly 2.5 over the same period. After adjustment for age according to age structure of China population in 1982, ASMR of lung cancer doubled during 1990-2005. Although it declined slightly during 2011-2013, ASMRs of lung cancer among males and females were 3 and 6 times of that in rural areas of China, respectively (Fig. 3). Compared with rural areas of China, lung cancer deaths occurred at younger ages in Xuanwei. Lung cancer mortality probability in young and middle-aged populations (45Q15 of lung cancer) was significantly higher than that in rural areas of China. The 45Q15 for males was 3 to 5 times of that in rural areas nationally and it was 7-9 times for females (Fig. 4).

\subsection{Regional distribution of lung cancer mortality rates}

A remarkable difference can be seen in mortality rates of lung cancer in varied communities with the highest mortality rate in all communities more than 20 times of the lowest. High-mortality areas were mainly in Labin, Longchang, Wanshui and Shuanglong, while low-mortality areas were mainly in Reshui, Wenxing and Wude. Our results show that the gap widened between the ASMR of lung cancer in each community in Xuanwei and that in rural areas of China (Fig. 5).

During 1990-2005, high-mortality areas of lung cancer $\left(\right.$ ASMR $\left.>80 / 10^{5}\right)$ expanded from areas with the highest ASMR of lung cancer (Laibing, Rocheng) mainly to their surrounding areas and those in their southeast. The ASMR in areas with the highest
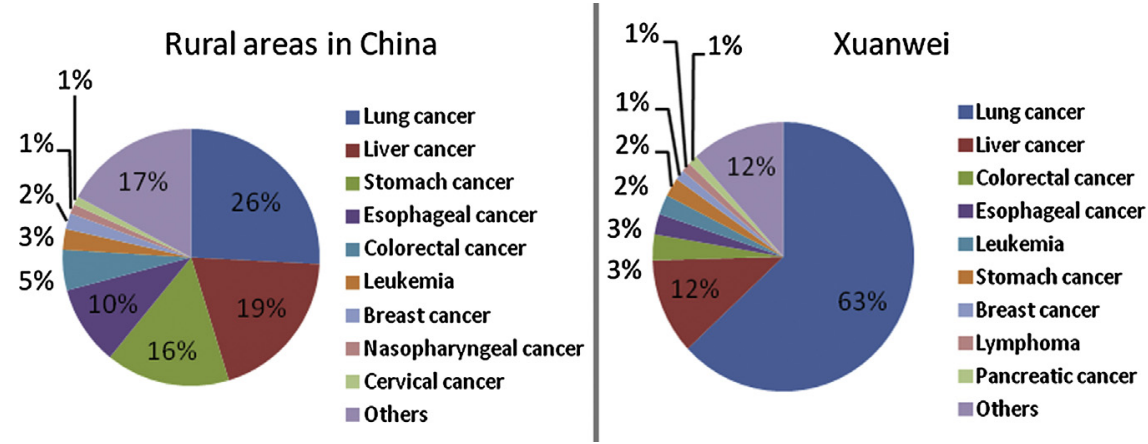

Fig. 2. Constituent of deaths due to cancers in Xuanwei and rural areas in China during 2011-2013. 


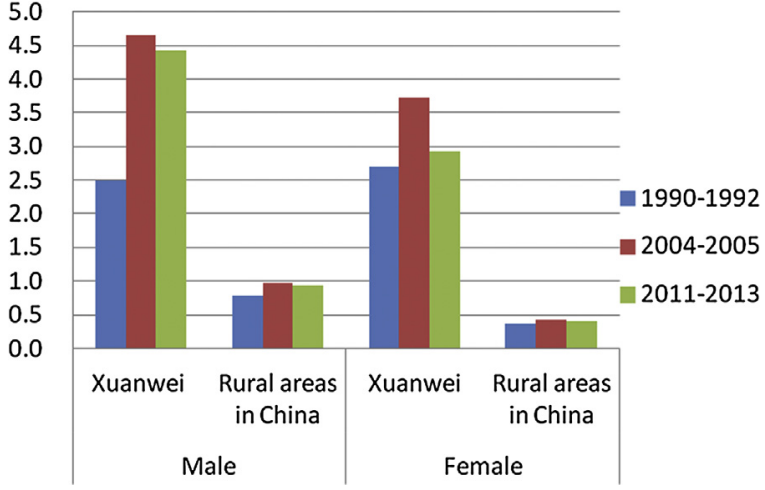

Fig. 4. 45Q15 of lung cancer in Xuanwei and rural areas of China during 1990-2013 (\%).

mortality of lung cancer reached over 160/105 . During 2011-2013, high-mortality areas of lung cancer display a slight contraction, but part of them in southwest, south and southeast remained high mortality of lung cancer (ASMR between $80 / 10^{5}$ and $160 / 10^{5}$ ). The ASMRs in original high-mortality areas of lung cancer still kept over $160 / 10^{5}$. In a few communities, such as Tangtang and Geyi, the ASMRs of lung cancer declined during 2011-2013. The areas with high mortality of lung cancer of females were more regionally concentrated than that of males. ASMR of lung cancer in each community and corresponding confidence intervals can be seen in Table A.4 in appendices.

\section{Discussion}

In Xuanwei, deaths from lung cancer and chronic respiratory disease (COPD) were several times those reported in rural areas in China, and these two types of diseases accounted for more than $30 \%$ of all deaths. The mortality of lung cancer kept increasing during past two decades with a more rapid rate than rural areas of China. Mortality of lung cancer in females approached that in males. High-mortality areas of lung cancer expanded from areas with the highest mortality of lung cancer to their surrounding areas and those in southeast. The areas with high mortality of lung cancer in females were more regionally concentrated than that in males. The ASMRs in low-mortality areas of lung cancer increased with a similar rate to rural areas of China.

The stove improvement project conducted in Xuanwei was effective $[9,10]$ targeting the indoor air pollution. In a cohort study conducted in Xuanwei by Barone-Adesi, et al. (1976-1996), 73.3\% of all participants were lifelong users of smoky coal and $87.3 \%$ of them have ever improved their stoves [5]. In an epidemiological study conducted in Xuanwei and nearby coal-producing areas in $2007,84.59 \%$ and $83.78 \%$ of all participants used smoky coal before and after the age of 20 , respectively. The annual average consumption of smoky coal has been reported to be 2.83 and 2.65 tons, also $80.8 \%$ and $22.8 \%$ of participants use unvented stoves before and after 20 years old, respectively [27]. In our ecological studies conducted in 6 communities of Xuanwei in 2014, 80.5\% and 80.1\% households used smoky coal ten years ago and at present, respectively. The annual average consumption is 4.9 and 2.4 tons, also $12.6 \%$ and $3.8 \%$ of households used unvented stoves ten years ago and at present, respectively (to be published). Thus, the proportion
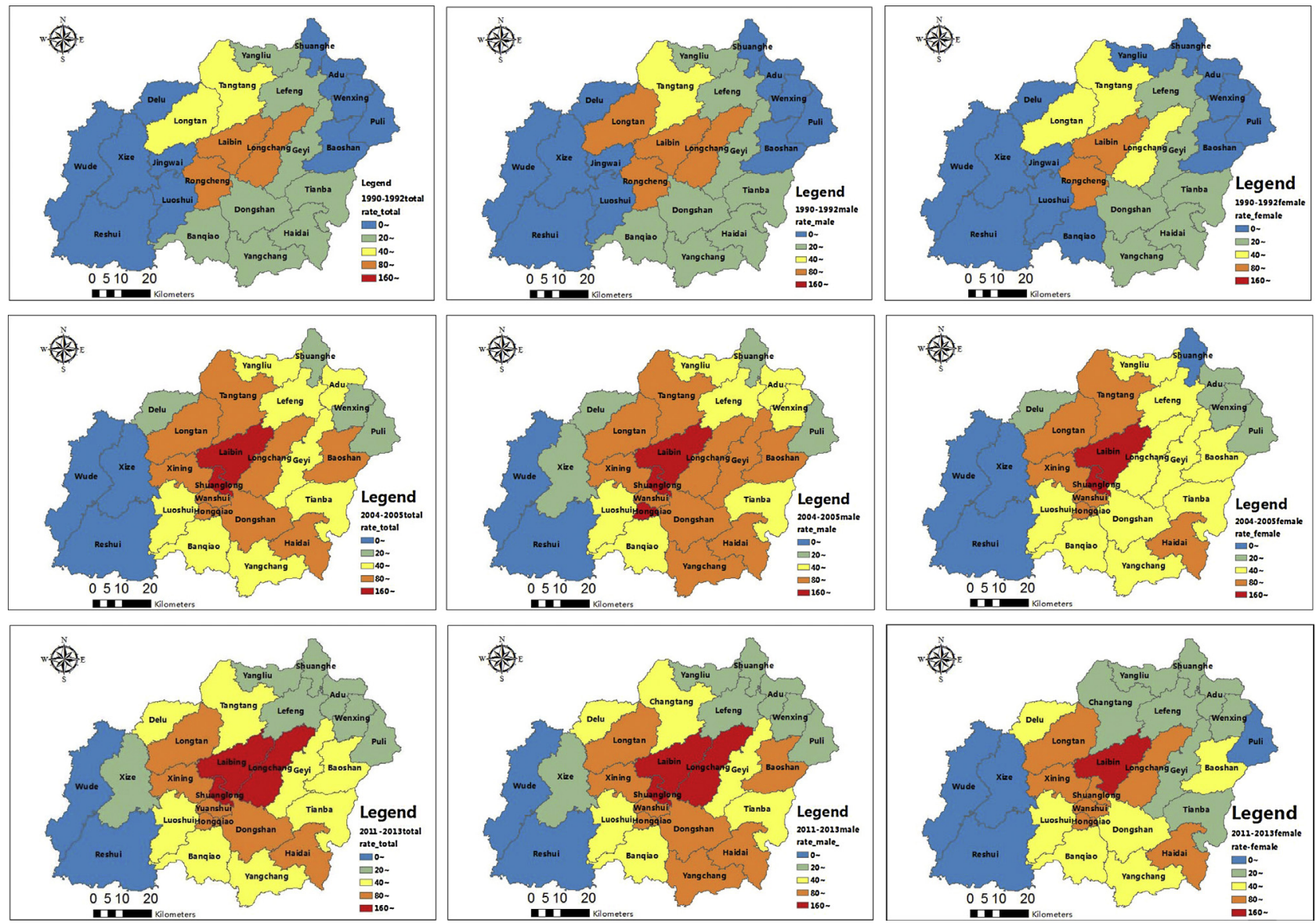

Fig. 5. ASMR of lung cancer in each community of Xuanwei during 1990-2013 (1/10 $)$. 
of households using smoky coal does not change significantly, while the consumption of smoky coal and the use of unvented stoves declined dramatically during past three decades.

According to previous studies, smoking was weakly associated with high mortality of lung cancer in Xuanwei $[3,28]$. Smoking rate did not vary significantly in different communities, however significant difference existed in mortality of lung cancer regionally [3]. On the other hand, smoking rates among males in two studies mentioned above were $92.3 \%$ and $85.5 \%$ respectively, and in our ecological studies it was $72.2 \%$, which were much higher than rural areas of China (56.1\%) [5,27,29]. The smoking rate among females was very low. The adverse effects of smoking on lung cancer in Xuanwei were more apparent as residents switch to cleaner fuels [10].

Rationally, after the removal of the main risk factors, lung cancer mortality among young and middle-age populations should decline. However, this change has not occurred and the lung cancer mortality rate in Xuanwei remained much higher than that in rural areas of China. Additionally, the characteristics of regional concentration of lung cancer mortality did not change. Therefore, household air pollution cannot completely explain the high mortality of lung cancer in Xuanwei. There are several possible reasons for this discrepancy. First, the stove improvement project was not effectively implemented. Residents' exposure to risk factors did not decline significantly, although most stoves have been improved, and the consumption of smoky coal has declined. According to local records, given government funding of stove improvement, some residents did not improve their stoves until their diagnoses of respiratory disease. Additionally, some interventions on risk factors are difficult to implement, such as outdoor air pollution and family history of disease [30,31]. Second, transition of mortality rate of lung cancer did not occur because of an accumulation effect of risk factors, although they have been controlled. Due to the accumulation effect of smoking, risk for lung cancer among ex-smokers is still higher than that in non-smokers, given the declined risk after quitting smoking [32]. Similarly, our results indicate the accumulation effect of indoor air pollution on lung cancer. Third, new risk factors for lung cancer have occurred during past decades or the changed effects of original risk factors on lung cancer. A study indicated the potential association between the location of the power plant, concentration of chemical factories, wind direction and lung cancer [33]. Also, smoking showed stronger effects on lung cancer after improvement of stoves and fuels in Xuanwei as mentioned.

However, there are some limitations in our study. Since Xuanwei is a poor mountain area, older patients were diagnosed at a lower level of medical institutions compared to rural areas in China $[11,13,24]$. And local DRS needs to be improved as we found local medical workers cannot well understand the meaning of evidence of diagnosis on death certification. The detail conditions in lung cancer diagnoses were also discussed elsewhere [27]. Since 1970 s, a series of studies have been conducted in Xuanwei focusing on high mortality rates due to lung cancer. Most of them have been conducted in areas with high mortality and have targeted domestic combustion of coals, but exposure to other risk factors among broader populations has seldom been assessed. In light of high mortality of lung cancer even after the control of main causes, further comprehensive studies should be conducted to implement effective interventions. More specifically, study areas should be expanded to low-mortality and medium-mortality areas that are relatively far away from city center. Additionally, studies should be conducted involving more risk factors from the perspective of varied disciplines, including behavioral and outdoor environmental risk factors. Finally, interactions between different risk factors should be taken into consideration.

\section{Conflicts of interest}

The authors have no conflicts or financial disclosures to make.

\section{Acknowledgments}

This work was supported by China Medical Board Grant on CMBCP in Burden of Diseases in China (12-107).

\section{Appendix A. Supplementary data}

Supplementary data associated with this article can be found, in the online version, at http://dx.doi.org/10.1016/j.lungcan.2015.08. 006.

\section{References}

[1] Office of Research for cancer prevention, Ministry of Health. Study on Cancer Mortality in China. People's Medical Publishing House. Beijing, 1979.

[2] J.L. Mumford, X.Z. He, R.S. Chapman, et al., Lung cancer and indoor air pollution in Xuan Wei, China, Science 235 (4785) (1987) 217-220.

[3] X.Z. He, W. Chen, Z.Y. Liu, R.S. Chapman, An epidemiological study of lung cancer in Xuan Wei County, China: current progress. Case-control study on lung cancer and cooking fuel, Environ. Health Perspect. 94 (1991) 9-13.

[4] Q. Lan, et al., Variation in lung cancer risk by smoky coal subtype in Xuanwei, China, Int. J. Cancer 123 (2008) 2164-2169.

[5] F. Barone-Adesi, R.S. Chapman, D.T. Silverman, X. in, C. uanwei, et al., Risk of lung cancer associated with domestic use of coal in Xuanwei, China: retrospective cohort study, BMJ 345 (2012) e5414.

[6] R.S. Chapman, J.L. Mumford, D.B. Harris, Z.Z. He, W.Z. Jiang, R.D. Yang, The epidemiology of lung cancer in Xuan Wei, China: current progress, issues, and research strategies, Arch. Environ. Health 43 (1988) 180-185.

[7] G.S. Downward, W. Hu, D. Large, et al., Heterogeneity in coal composition and implications for lung cancer risk in Xuanwei and Fuyuan counties, China, Environ Int. 68C (2014) 94-104, 2014-04-07.

[8] J.L. Mumford, C.T. Helmes, X.M. Lee, J. Seidenberg, S. Nesnow, Mouse skin tumorigenicity studies of indoor coal and wood combustion emissions from homes of residents in Xuan Wei, China with high lung cancer mortality, Carcinogenesis 11 (1990) 397-403.

[9] O. Lan, R.S. Chapman, D.M. Schreinemachers, L. Tian, X. He, Household stove improvement and risk of lung cancer in Xuanwei, China, J. Natl. Cancer Inst. 94 (2002) 826-835.

[10] C. Kim, R.S. Chapman, W. Hu, et al., Smoky coal, tobacco smoking, and lung cancer risk in Xuanwei, China, Lung Cancer J. Iaslc. 84 (2014) 31-35.

[11] Ministry of Health P.R.C. The third retrospective sampling survey report of causes of death. Peking Union Medical College Press. Beijing, 2012.

[12] Y. Xiao, Y. Shao, X. Yu, G. Zhou, The epidemic status and risk factors of lung cancer in Xuanwei City, Yunnan Province, China, Front. Med. 6 (4) (2012) 388-394.

[13] National Office of Research on Cancer Prevention. Survey on cancer mortality in China (1990-1992). Peoples Medical Publishing House. Beijing, 2008.

[14] Population Census Office under the State Council and Department of Population Statistic, State Statistical Bureau, People's Republic of China. Tabulation on the 1990 Population Census of the People's Republic of China. China Statistical Publishing House. Beijing, 1993.

[15] Population Census Office under the State Council and Department of Population, Social, Science and Technology Statistics, National Bureau of Statistics of China. Tabulation on the 2000 Population Census of the People's Republic of China. China Statistics Press. Beijing, 2002.

[16] Population Census Office under the State Council and Department of Population and Employment Statistics, National Bureau of Statistics. Tabulation on the 2010 Population Census of the People's Republic of China. China Statistics Press. Beijing, 2012.

[17] Xuanwei Municipal Committee and Xuanwei Government. Year Book of Xuanwei. De Hong Min Zu Press. Yunnan, 2012.

[18] Chen Gongbo, Huang Hecang, Ma Xiangyun, et al. Investigation on mis-reported deaths in Xuanwei, Yunnan Province, during 2011-2013. Zhonghua Yu Fang Yi Xue Za Zhi. 2015;6:541-5. (in Chinese).

[19] Dept. of Control Disease of MOPH and Chinese Academy of Preventive Medicine. 1995 Annual Report on Chinese Diseases Surveillance. People's Medical Publishing House. Beijing, 1997.

[20] Manual of the International Statistical Classification of Diseases, Injurues and Causes of Death, Ninth Revision. Geneva: World Health Organization, 1977.

[21] World Health Organization. International Classification of Diseases and related health problems - Tenth Revision (ICD 10). Geneva: WHO, 1992

[22] Z. Fu, J. Dong, B. Guo, et al., Study on adjustment of pulmonary heart disease, J. Hyg. Res. (Wei Sheng Yan Jiu) 2 (1988) 50-51 (in Chinese).

[23] National Office of Research on Cancer Prevention, National Center of Cancer registry and Disease Prevention and Control Bureau of Ministry of Health P.R.C. The China Report on Cancer Mortality. People's Medical Publishing House. Beijing, 2010. 
[24] Center of Non-communicable Disease Control and Prevention of China CDC Death Surveillance Dataset of Diseases Surveillance Point System. Popular Science Press. Beijing, 2013.

[25] G. Richard, A. Feachem, T. Kjellstrom, J. Christopher, L. Murry, et al., The Health of Adults in the Developing World. Published for the World Bank Oxford University Press, New York, 1992.

[26] P. Armitage, G. Berry, Statistical Methods in Medical Research, 2nd ed., Scientific Publications, Boston, 1987.

[27] J. Li, Y. Zhang, Y. Li, et al., Descriptive study on the epidemiology of lung cancer in coal-producing area in Eastern Yunnan, China, Chin. J. Lung Cancer 14 (2011) 107-119 (in Chinese).

[28] X.Z. He, Q. Lan, R.D. Yang, R.F. Li, C.F. Huang, An overview of studies on risk factors for lung cancer in Xuanwei, China (1979-1993), J. Hyg. Res. (Wei Sheng Yan Jiu) 24 (4) (1995) 203-206 (in Chinese).
[29] China Center for Disease Control and Prevention. Global Adult Tobacco Survey (GATS) China 2010Country Report. San Xia Press. Beijing, 2011.

[30] J. Lv, R. Xu, G. Wu, et al., Indoor and outdoor air pollution of polycyclic aromatic hydrocarbons (PAHs) in Xuanwei and Fuyuan, China, J Environ Monit. 11 (2009) 1368-1374

[31] H.D. Hosgoodiii, R.S. Chapman, X. He, et al., History of lung disease and risk of lung cancer in a population with high household fuel combustion exposures in rural China, Lung Cancer J. Iaslc. 81 (2013) 343-346.

[32] R. Peto, S. Darby, H. Deo, P. Silcocks, E. Whitley, R. Doll, Smoking, smoking cessation, and lung cancer in the UK since 1950: combination of national statistics with two case-control studies, BMJ 321 (2000) 323-329.

[33] Y. Cao, H. Gao, Prevalence and causes of air pollution and lung cancer in Xuanwei City and Fuyuan County, Yunnan Province, China, Front Med. 6 (2012) 217-220. 